This paper is published as part of a CrystEngComm theme issue on:

\title{
Mechanochemistry and cocrystals
}

\author{
Guest Editors: \\ Stuart James - The Queen's University of Belfast, UK \\ Ashwini Nangia - University of Hyderabad, India
}

Published in issue 3, 2009 of CrystEngComm
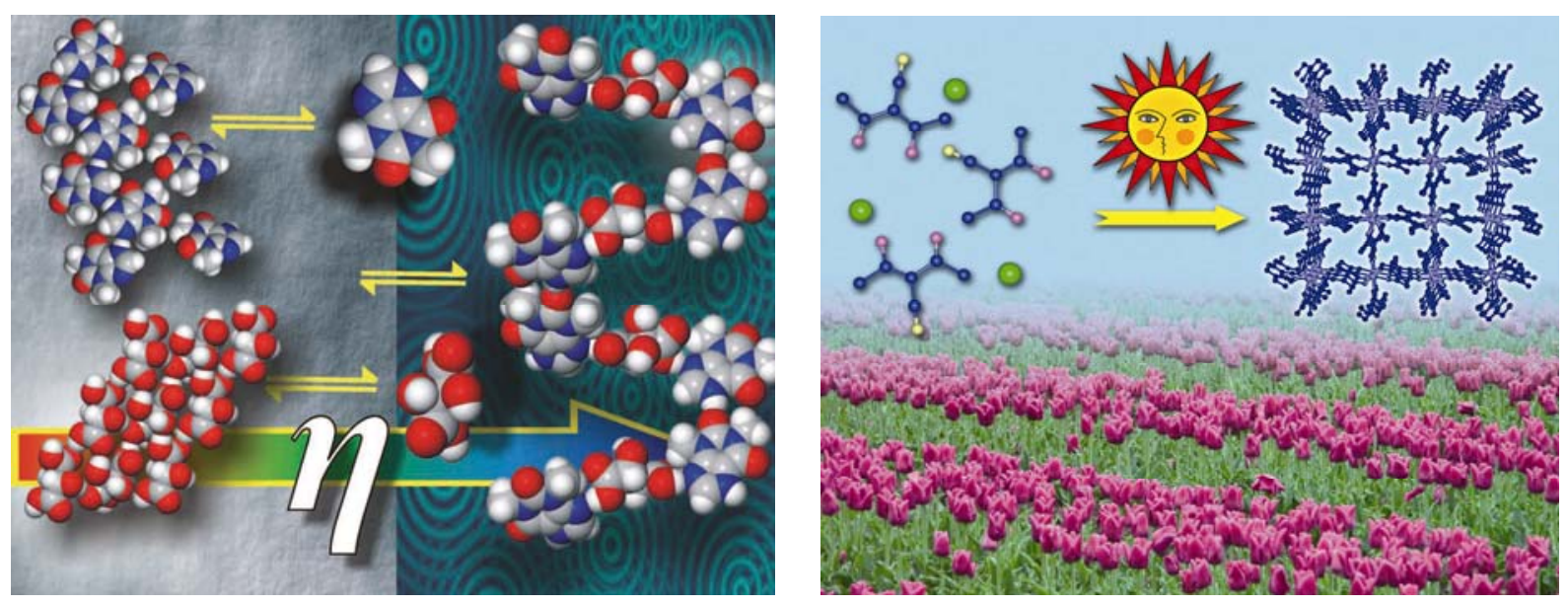

Images reproduced with permission of Tomislav Friscic (left) and Reiko Kuroda (right)

Papers published in this issue include:

Mechanochemistry: the varied applications of mechanical bond-breaking

Gerd Kaupp, CrystEngComm, 2009

DOI: $\underline{10.1039 / \mathrm{b} 810822 \mathrm{f}}$

Nonlinear effects in proline-catalysed aldol reactions under solvent-free conditions based on the ternary phase behaviour of scalemic proline Angelika Bruckmann, Belén Rodríguez and Carsten Bolm, CrystEngComm, 2009

DOI: $\underline{10.1039 / \mathrm{b} 821415 \mathrm{~h}}$

The role of solvent in mechanochemical and sonochemical cocrystal formation: a solubilitybased approach for predicting cocrystallisation outcome

Tomislav Friscic, Scott L. Childs, Syed A. A. Rizvi and William Jones, CrystEngComm, 2009

DOI: $10.1039 / \mathrm{b} 815174 \mathrm{a}$

Annealing assisted mechanochemical syntheses of transition-metal coordination compounds and co-crystal formation

Reiko Kuroda, Jun Yoshida, Asao Nakamura and Shin-ichi Nishikiori, CrystEngComm, 2009

DOI: $10.1039 / \mathrm{b} 819701 \mathrm{f}$

The mechanochemical synthesis of podand anion receptors

Adam N. Swinburne and Jonathan W. Steed, CrystEngComm, 2009

DOI: $\underline{10.1039 / \mathrm{b} 817067 \mathrm{c}}$

Visit the CrystEngComm website for more cutting-edge crystal engineering research www.rsc.org/crystengcomm 


\title{
SFM study of the surface of halogen-bonded hybrid co-crystals containing long-chain perfluorocarbons $\dagger$
}

\author{
Giuseppe Maruccio, ${ }^{* a}$ Valentina Arima, ${ }^{a}$ Roberto Cingolani, ${ }^{a}$ Rosalba Liantonio, ${ }^{b}$ Tullio Pilati, ${ }^{c}$ \\ Ross Rinaldi ${ }^{a}$ and Pierangelo Metrangolo*b
}

\author{
Received 26th August 2008, Accepted 21st November 2008 \\ First published as an Advance Article on the web 22nd December 2008 \\ DOI: $10.1039 / \mathrm{b815816a}$
}

\begin{abstract}
Different scanning force microscopy (SFM) techniques were employed to investigate the structure and composition of the fundamental crystal faces of prototype halogen-bonded co-crystals of long-chain perfluorocarbons. These crystals were found to show surfaces with well-defined ledges formed by intersecting crystal planes having different chemical compositions with the perfluorocarbons (PFCs) covering the largest area of the crystal as a reminiscence of the strong segregation observed in the bulk crystal structure.
\end{abstract}

\section{Introduction}

Perfluorocarbons (PFCs) represent a very interesting and stimulating class of chemicals, with large impact in numerous fields (from materials science ${ }^{1}$ to supramolecular chemistry ${ }^{2}$ and biomedical engineering ${ }^{3}$ ) because of their unusual properties (such as very low surface tensions, dielectric constants, and refractive indexes, alongside high densities, viscosities, and gas solubilities ${ }^{4}$ ), which are due to their chemical structures and the weak intermolecular interactions they typically give rise to. Thanks to these properties, PFCs are commonly employed in a wide variety of technological areas (e.g. surfactants in supercritical solvents ${ }^{5}$ or anticorrosive and antifriction components, water repellents, sliding agents in paints, coatings, and polymer technology ${ }^{6}$ ). On the other side, the same unique properties also account for the poor affinity the PFCs have for hydrocarbons (HCs), water, and salts (both organic and inorganic), also known as fluorophobic effect. This fact represents a major limitation if the PFC-HC recognition process is pursued to the point of triggering the self-assembling of the two species into hybrid structures like co-crystals. Specifically tailored patterns of intermolecular interactions between PFCs and HCs are thus required.

Recently, some of us showed the effectiveness of an innovative protocol based on "halogen bonding" (XB), ${ }^{7}$ which is defined as any attractive interaction involving halogens as electrophilic species, for specifically involving PFCs in intermolecular

${ }^{a}$ National Nanotechnology Laboratory of CNR-INFM, Scuola Superiore Isufi, University of Salento, Via per Arnesano, 73100, Lecce, Italy. E-mail: giuseppe.maruccio@unile.it; Fax: +39 0832 298238; Tel: +39 0832298241

${ }^{b}$ Laboratory of Nanostructured Fluorinated Materials (NFMLab), Department of Chemistry, Materials, and Chemical Engineering "G. Natta", Politecnico di Milano, Via L. Mancinelli 7, 20131, Milan, Italy. E-mail: pierangelo.metrangolo@polimi.it; Fax: +3902 2399 3180; Tel: +3902 23993041

${ }^{c}$ C.N.R.-Institute of Molecular Sciences and Technologies, University of Milan, Via C. Golgi 19, 20133, Milan, Italy

$\dagger$ Electronic supplementary information (ESI) available: Histogram for type A curves showing the distribution of adhesion forces. See DOI: $10.1039 / \mathrm{b} 815816 \mathrm{a}$ recognition processes with $\mathrm{HCs}$ and/or organic and inorganic salts (OSs and ISs, respectively). This has opened new perspectives in the manipulation and control of PFCs' aggregation phenomena. Thanks to the electron withdrawing ability of fluorine atoms, halo-PFCs are particularly well-suited for giving rise to strong halogen bonds. The XB-induced self-assembly of iodo- and bromo-PFCs with HC electron-donor modules was already demonstrated to be an efficient strategy in producing PFC-HC co-crystals ${ }^{8}$ with applications in various fields (such as liquid crystals ${ }^{9}$ and non-covalent fluorous coatings $\left.{ }^{10}\right)$.

Moreover, the approach of involving haloperfluoroalkanes in co-crystal formation with $\mathrm{XB}$-acceptors has disclosed new perspectives in the study of long perfluoroalkyl chains with analytical techniques, e.g. single-crystal X-ray diffraction methods, which were only occasionally used before our studies. In fact, while growing single crystals of long-chain perfluoroalkanes is very difficult, due to the poor intermolecular interactions PFCs give rise to, most of the reported halogenbonded PFC-HC materials are crystalline, demonstrating the general ability of halo-PFCs to behave as robust tectons in XB-based crystal engineering. ${ }^{7}$

Nowadays, scanning force microscopy (SFM) techniques are becoming more and more employed in the structural characterization of organic crystal surfaces and as tools for elucidating the crucial features of the crystallization process. ${ }^{11-13}$ In this article, we report the first characterization by different SFM techniques (atomic force microscopy (AFM), lateral force microscopy (LFM), surface potential microscopy (SP), and force spectroscopy (FS)) of a prototype halogen-bonded crystalline system containing long perfluoroalkyl chains. ${ }^{14}$ All these analytical techniques have been jointly applied to obtain structural and surface compositional information, and to investigate the properties of these hybrid materials at the nanoscale level. The hybrid co-crystals studied were found to show surfaces with well-defined ledges formed by intersecting crystal planes having different, identifiable chemical composition. In particular, the perfluorinated modules cover the major part of the surface of the crystal, which is reminiscent of the strong segregation between PFC and HC modules that characterizes the bulk crystal structure. 
We expect this study to impact the rational design of halogenbonded PFC-HC systems, leading to subsequent synthesis of novel hybrid co-crystals exposing engineered surfaces and exhibiting predictable functional properties.

\section{Experimental}

Crystals of the three-component supramolecular complex $\mathbf{5}$ (Fig. 1a) were obtained on isothermal evaporation under diffusion conditions of an ethanol solution containing equimolar amounts of 4,7,13,16,21-24-hexaoxa-1,10-diazabicyclo[8,8,8]hexacosane (1, cryptate Kryptofix ${ }^{\circledR} 222$ ), potassium iodide (2) and $\quad 1,1,2,2,3,3,4,4,5,5,6,6,7,7,8,8$-hexadecafluoro-1,8-diiodooctane (4). A multistep supramolecular synthesis ${ }^{15}$ occurs, where two different interactions $\left(\mathrm{K}^{+}\right.$binding by K.2.2.2 1 and $\left.\mathrm{I}^{-} \cdots \mathrm{I}-\mathrm{PFC} \mathrm{XB}\right)$ prevail over other possible interactions $(e . g$. $\mathrm{HC}-$ $\mathrm{N} \cdots \mathrm{I}-\mathrm{PFC} \mathrm{XB})$ and work in a hierarchical fashion. Alternatively, crystals of 5 can be obtained by dissolving in ethanol, in a vial of clear borosilicate glass at room temperature, equimolar amounts of 3 (cryptate Kryptofix ${ }^{\circledR} 22 \subset$ KI formed by $\mathbf{1}$ and 2 ) and $\mathbf{4}$, closing the open vial in a cylindrical wide-mouth bottle containing paraffine oil and allowing the solvent to diffuse at rt. After a period of 1-5 days, colorless crystals precipitated from the solution (for a detailed description and single crystal X-ray characterization of the halogen-bonded system 5 , see ref. 14).

Scanning probe imaging was performed with a commercial scanning probe microscope (Veeco Thermomicroscope CPresearch II). An amplitude modulation technique was used to image topography, while the electrostatic force between tip and sample was exploited for simultaneously sensing the surface potential by applying to a metal-coated tip an AC voltage in addition to a DC voltage. Varying this DC voltage in order to compensate the total electrostatic force to zero, a feedback loop allowed to directly measure the surface potential. ${ }^{16}$ LFM imaging and FS were performed in air using the same microscope in contact mode. Silicon nitride cantilevers were employed in FS studies, which enable quantitative investigation of nanoscale forces after a proper calibration. All these SFM techniques are able to distinguish regions exposing different chemical groups.

\section{Results and discussion}

\section{Crystal description}

Our attention was focused on the ternary supramolecular complex $\mathbf{5}$ (Fig. 1a), ${ }^{\mathbf{1 4}}$ given by $\mathbf{4}$ with the cryptate Kryptofix ${ }^{\circledR} 222$ $\subset$ KI 3. This complex crystallizes with trigonal symmetry in the $P \overline{3}$ space group and forms large hexagonal or triangular thin plates (Fig. 1e) having their largest faces perpendicular to the $c$ crystallographic axis. The bulk crystal structure of $\mathbf{5}$ is characterized by alternating supercations' $\left(\mathrm{K} \cdot 2.2 .2 \subset \mathrm{K}^{+}\right)$and super-

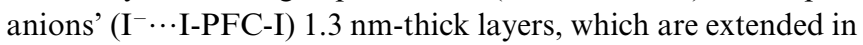
the (001) crystallographic plane (Fig. 1b). The superanions' layers consist of interpenetrated infinite [6,3] networks (Fig. 1c) assembled thanks to $\mathrm{I}^{-} \cdots \mathrm{I}$-PFC-I XB. Iodide ions, which work as pyramidal tridentate electron donors, are the nodes of the nets, while diiodo-PFCs 4, which behave as bidentate electron acceptors, connect the nodes. The ionic pair is fully separated as

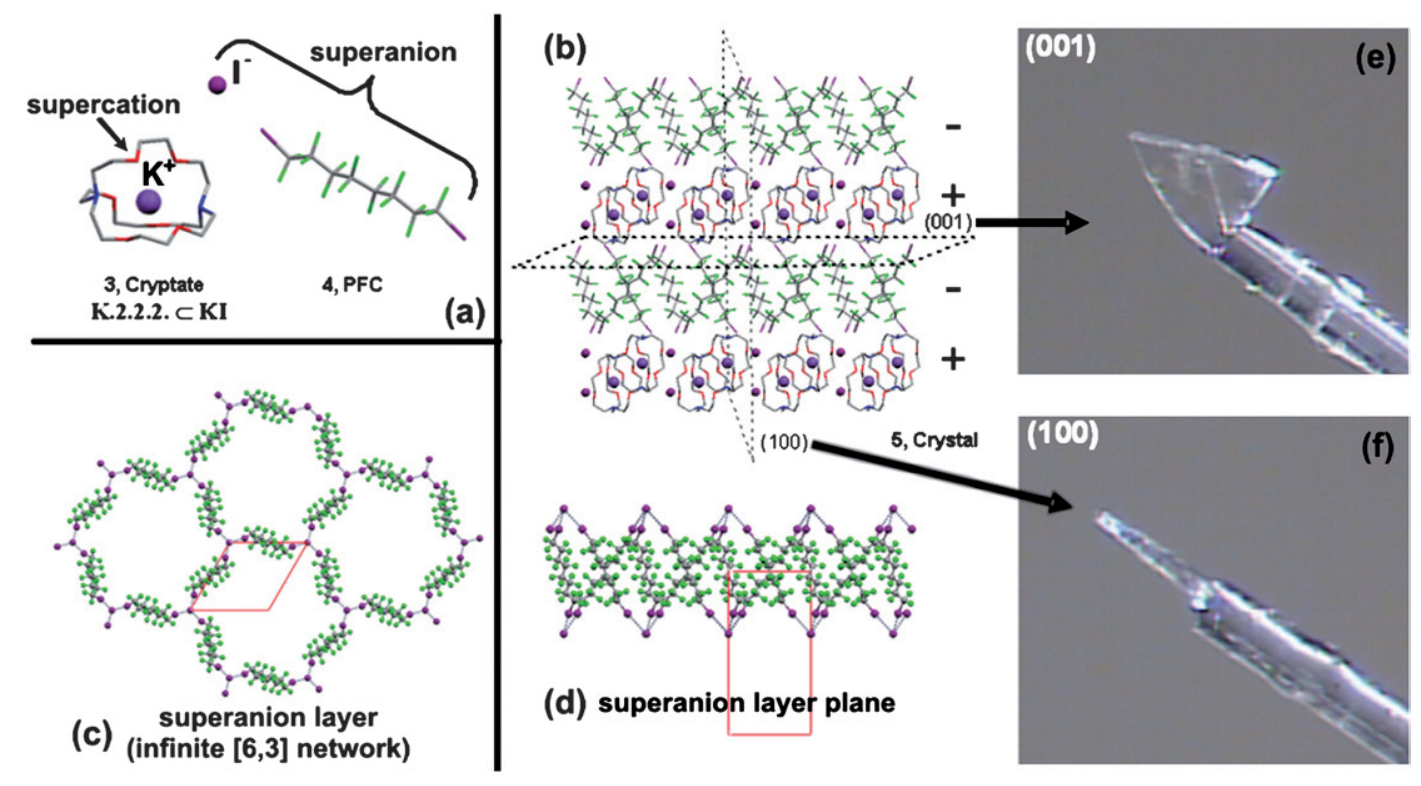

Fig. 1 (a) Asymmetric unit of the structure of the co-crystal 5 formed by the cryptate 3 (K.2.2.2 $\subset \mathrm{KI}$ ) and 1,8-diiodoperfluorooctane 4 . (b) Bulk crystal structure of the co-crystal 5 viewed down the $a$ crystallographic axis. Reminiscent of the unique phase properties of PFC derivatives, the cryptate and the perfluorinated modules give rise to a remarkable segregation in the crystal packing. Alternating PFC-HC/IS layers are formed ( $c a$. $1.3 \mathrm{~nm}$ thick), which develop along the $c$ crystallographic axis. Two (001) PFC-HC/IS bilayers are reported, which are separated by a plane with horizontal dashed outlines. (c) and (d) The 6.3 network of superanions ( $\mathrm{I}^{-} \cdots \mathrm{I}-\mathrm{PFC}$ ) viewed down the $c$ (c) and $a$ (d) crystallographic axes. Iodide ions are the nodes of the network and connect three different diiodoperfluoroalkanes 4 through XB. The strongly anisotropic XB along with the stiff character of the PFC units force the growth of the layers in the [001] zone of the crystal. Colours are as follows: $\mathrm{C}$, grey; N, blue; O, red; F, green; K, violet; and I, purple. XB is represented as dashed lines. (e) Optical microscopy image of the (001) face of a $0.40 \times 0.27 \times 0.05 \mathrm{~mm}^{-3}$ triangular-shaped crystal of the supramolecular complex 5 mounted over a glass capillary. (f) Optical microscopy image of the (100) face of the same crystal. 
the shortest $\mathrm{K}^{+} \cdots \mathrm{I}^{-}$distance is $7.681 \AA$. Assuming thermodynamic control, the relative areas covered by the specific crystal faces are inversely proportional to their relative surface energies, which depend on the exposed molecular functionalities. By definition, the lowest energy surface is the one in which the weakest bonds are truncated, since this minimizes the energetic penalty associated with the lack of bonding at this plane. ${ }^{11}$ It could, thus, be expected that the electrostatic interactions between supercations and superanions will be truncated. On the other hand, the strong ${ }^{17}$ anisotropic $\mathrm{I}^{-} \cdots$ I-PFC-I interactions drive the crystal growth of $\mathbf{5}$ preferentially in the superanions' layer plane (Fig. 1d), which is perpendicular to the $c$ axis. In addition, during crystal growth, the charged surfaces (covered by superanions and supercations) are continuously neutralized by adsorption of couterions from solution. Such process is expected to slow down the growth rate of the surface, causing the dominant face to have this orientation. Both these characteristics should tend to favor the formation of well-defined ledges on the crystal surface, whose structure (given the trigonal symmetry of 5) can be surmised (in absence of a significant reconstruction) from the analysis of the bulk crystal structure. We can thus foresee the (001) surface to have a single-component composition (either the cryptate 3 or the PFC 4), while the [001] zone composition corresponds to the PFC-HC/IS layers, which alternate along the $c$ crystallographic axis. Identifying the specific functional groups exposed at the different crystal faces of $\mathbf{5}$ would greatly contribute to better understanding of the crystal growth of these hybrid materials, impacting the rational design of halogen-bonded PFC-HC crystals exposing engineered functional surfaces with tailored properties.

For this purpose, we characterized the crystals through different SFM techniques. Many examples on the effectiveness of these techniques in the characterization of phase-separated mixed PFC-HC systems are reported in the literature. ${ }^{18-20}$ Here we extended their use to the study of hybrid PFC-HC/IS halogenbonded co-crystals for obtaining structural and compositional information on their surfaces. In fact, the ability of $\mathrm{SP}^{21}$ and $\mathrm{LFM}^{22}$ to detect terminal group differences enables the investigation of the ordered growth of crystals' faces and the correlation of topographic and compositional features in order to identify faces with different chemical composition.

\section{SPM results}

Tapping AFM in combination with SP mapping was first used for the simultaneous visualization of the topographical features and the local surface potential $\left(V_{\mathrm{S}}\right)$ at the fundamental crystal surfaces. Specifically, we started investigating the surface of plate-like crystals of $\mathbf{5}$, as formed after the precipitation from an ethanol solution and oriented in order to expose their largest (001) surface (Fig. 1e). Fig. 2 reports the topographic (a) and SP images (b) collected over a $1.5 \mathrm{~mm}$-large crystal. We observed alternating large and narrow terraces with a minimal separation roughly corresponding to the $c$ axis of the unit cell of $\mathbf{5 .}{ }^{23}$ The RMS roughness calculated on the crystalline terraces is typically around $2 \AA$ with occasional small clusters having lateral dimensions in the range of $150-200 \mathrm{~nm}$. The SP image (Fig. 2b) provides the first surface compositional information, thanks to the ability of SP microscopy to detect the potential on a surface. ${ }^{24}$

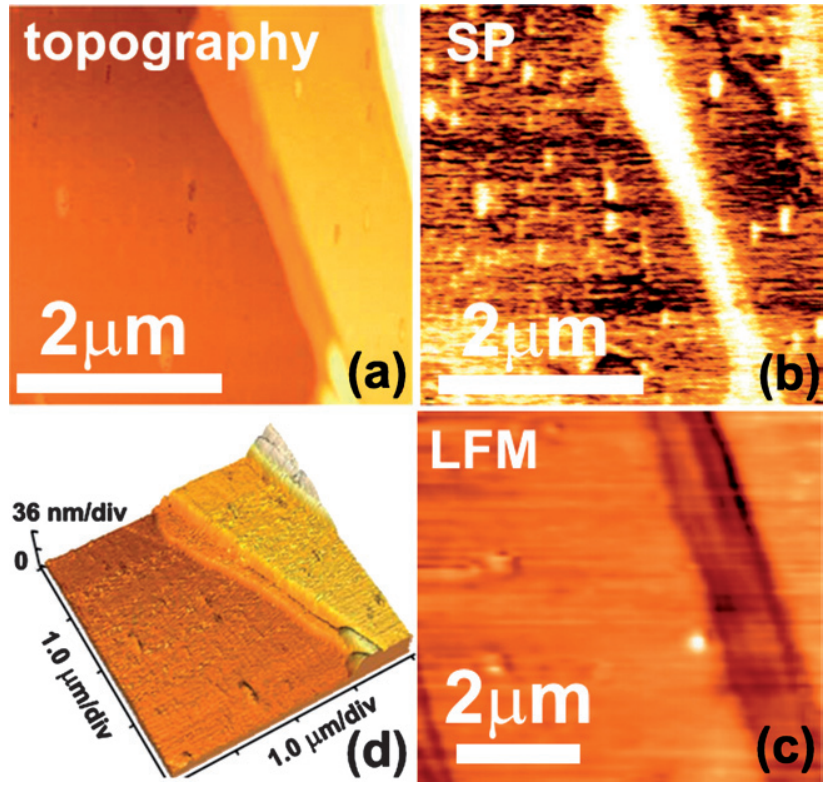

Fig. 2 (a) Constant amplitude topographic and (b) SP images recorded in air on the largest (001) surface of a $1.5 \mathrm{~mm}$ large crystal of 5 as precipitated from an ethanol solution. Well-defined crystalline terraces having different $V_{\mathrm{S}}$ values are visible. (c) LFM image recorded in air on the same crystallographic surface, confirming that two different molecular functionalities are exposed on this crystal surface. (d) 3D image of the crystal topography.

Alternating large "dark" and narrow "bright" regions were observed as a function of the low and high $V_{\mathrm{S}}$ values, respectively. The surface potential at the top of the bright regions is typically advanced by $25 \mathrm{mV}$ with respect to the dark ones, but this value should not be intended as quantitative since reliable measurements in air of the absolute difference of potentials are challenging (and beyond the aim of the present work). A strict correlation exists between the alternating terraces in the topography image and equipotential regions in SP mode. Large and narrow terraces correspond to dark and bright regions, respectively. It is worth noting that this correlation is not based on a variation of topography. As a consequence, we ascribe these results to surfaces with different chemical composition.

To provide complementary data, we investigated the same surface by LFM, which maps the lateral deformation of the cantilever during the scanning of the sample in contact mode. Since this deformation depends on the frictional force acting on the probe (measured over a localized contact area of a few square nanometers ${ }^{19}$ ), this technique is sensitive to chemical composition $^{19,25}$ and allows to distinguish components in multicomponents systems on the basis of their different frictional response. ${ }^{19}$ In good agreement with the SP results, we observed again alternated terraces with different friction (see Fig. 2c). In particular, large and narrow terraces correspond to high and low friction regions, respectively. Therefore, both SP and LFM data suggest that alternating domains exposing different molecular functionalities are present on the (001) surface of 5 .

These two regions, observed on the (001) surface of $\mathbf{5}$, can be correlated with the different chemical composition of its fundamental faces, as derived from the analysis of the bulk crystal 
structure of 5. Accordingly, we conclude the (001) crystal surface of 5 to have a single-module composition, either the cryptated salt 3 or the PFC 4. As a reference, in SP and LFM images of the [001] zone, on whose surface PFC-HC/IS are expected to alternate, we observed contrast only in correspondence with the clusters, at the spatial resolution achieved. Thus, further experiments with techniques other than SP and LFM (with a better spatial resolution) are required for probing this crystal zone.

To deeper understand the surface composition of $\mathbf{5}$ and directly investigate both the crystallographic (001) surface (with a single-module composition, Fig. 1e) and the [001] zone (expected to exhibit a two-module composition, Fig. 1f), FS was performed on high quality single crystals intentionally oriented in order to show these two fundamental faces. As a rule, we acquired a large number of curves (typically some tens), at different positions on a single surface and checked their reproducibility. All of the force curves reported have been acquired using the same tip, in order to exclude any influence of the tip radius on the measured adhesion force. Fig. 3a shows FS curves

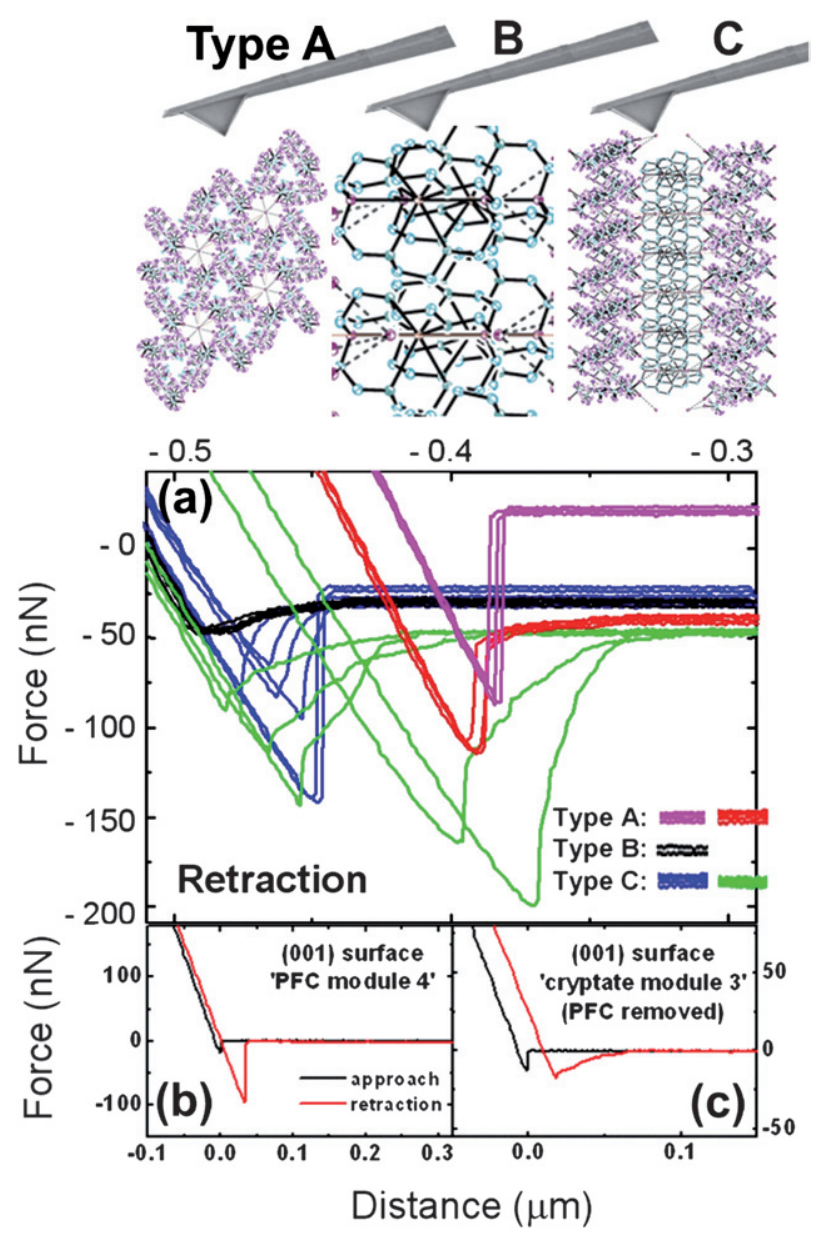

Fig. 3 (a) Five force-distance curves for each crystal, recorded in retraction at different points. It is noteworthy that while type A and type B curves are very reproducible in different scans, type C curves are strongly point-dependent, gradually switching from type A to type B behaviour and vice versa. (b)-(c) Force-distance curves recorded in approach and retraction on the (001) surface of 5, before (b) and after (c) vacuum removal of the PFC module 4. recorded on different crystal surfaces. Specifically, the violet and red curves (type A) were collected on the large terraces ("SP dark regions") of the (001) surface of two different crystals of $\mathbf{5}$. They are identical and perfectly reproducible in different scans and in different points of the surface (with a slight influence of humidity ${ }^{26}$ ), thus revealing again a homogeneous molecular composition of the examined areas of the (001) surface.

Exploiting the volatility of perfluorinated compounds, we then selectively removed the 1,8-diiodoperfluorooctane module 4 from the surface. The AFM force curves acquired on the (001) surface after this procedure (black curves in Fig. 3a, type B) differ greatly from the type $\mathrm{A}$ ones and always show low adhesion forces ascribable to a long-range attractive electrostatic force with a $20 \mathrm{~nm}$ Debye length ${ }^{27}$ (Fig. 3c). On the other hand, on the [001] zone of 5, we observed strongly surface-point dependent curves (green and blue curves in Fig. 3a, type C, from two different samples of 5). This is consistent with a surface exposing both modules (alternating 3 and 4), with the tip probing the local forces at the approach point, ${ }^{28}$ thus sensing a variable contribution from each module depending on the nearest molecular functionalities. Force spectroscopy (FS) curves acquired in approach mode showed no significant differences and no damage of the surface was ever observed after the FS measurements.

\section{Discussion}

The main point to understand for further advancing the rational design of these halogen-bonded PFC-HC crystals in order to exhibit functional/nanostructured surfaces with tailored properties is to unambiguously identify the molecular functionalities exposed on the different crystal faces. This is the field where SFM techniques can provide useful information for confirmation of crystal structure and compositional identification. ${ }^{11}$ Since large terraces exhibit a smaller SP value, we assign them to a surface exposing the perfluorinated modules of the superanion layer, while small terraces correspond to the superficial cryptated salt 3 module which results in a higher SP value since in this case the tip is closer to the supercation layer. The fact that PFCs are known as the solids with the lowest surface energies on earth ${ }^{6}$ strengthens the expectation that the presence of the PFC module 4 on the largest area of the (001) surface of 5 will contribute to the minimization of the overall surface energy of the crystal. Moreover, type A curves are strictly similar (large adhesion) to the ones that can be obtained on pure 1,8-diiodoperfluorooctane module 4 (not shown), supporting this assignment. On the other hand, we attribute the cryptate $\mathbf{3}$ module to be exposed on the narrow terraces, to whom type B curves correspond in FS experiments. In this respect, it is worth noting that the type $C$ force curves appear to gradually switch from type A to type B behaviour and vice versa depending on the analyzed point on the surface, supporting the two modules chemical composition at the [001] surface of the crystal 5.

Concerning the large adhesion forces measured in type A curves (typically $\approx 100 \mathrm{nN}$; Fig. $3 \mathrm{~b}$ ), at first glance this could seem an unexpected result for surfaces given by PFCs and their derivatives ${ }^{25,29-31}$ being typically strongly hydrophobic. However, FS curves acquired on the pure perfluoroalkane 4 exhibit the same shapes and adhesions (not shown). Thus, we speculate about a possible specific interaction between the tip and the 
surface. This possibility has been considered, for instance, to explain why the adhesion between a $\mathrm{Si}_{3} \mathrm{~N}_{4}$ tip and a silicon surface is stronger than what was found on PEI films of similar wettability. ${ }^{32}$ Specifically, in our case, we have a surface exposing iodo-terminated perfluoroalkanes, while all of the FS reports published so far concern $\mathrm{CF}_{3}$-terminated perfluoroalkanes. As a consequence, we have an array of XB-donor sites on the surface, while the tip material is $\mathrm{Si}_{3} \mathrm{~N}_{4}$. At first glance, a possible $\mathrm{XB}$ interaction between the tip $\mathrm{N}$ atoms, as XB-acceptor sites, and the iodine atoms on the surface seems plausible and would contribute to explaining the strong adhesion forces observed on regions covered by the 1,8-diiodoperfluorooctane module 4. A specific FS study of such surfaces with XB-acceptor groupfunctionalized tips is under current investigation and the results will be reported in due course.

Finally, the friction values have to be discussed. Since PFCs are employed as antifriction components in fluorine-containing lubricants, a reduction in friction could be expected on the fluorinated surfaces. However, Overney et al. reported a fourfold higher friction on fluorocarbons than on hydrocarbons, which they attributed, at least in part, to the greater stiffness and closer packing of the fluorocarbon moiety. ${ }^{19}$ In addition, in our case, the large adhesion forces observed on the large terraces on the (001) crystal surface found to expose the PFC $\mathbf{4}$ module are expected to result in higher lateral forces as compared to region exhibiting the cryptate $\mathbf{3}$ (where adhesion is low).

\section{Conclusions}

Four different SFM techniques (AFM, LFM, SP, and FS) were used to analyze, for the first time, the surface of the prototype halogen-bonded PFC-HC/IS hybrid supramolecular complex 5, which is characterized by strong segregation between the perfluorocarbon and the organic salt modules into alternating layers. We demonstrated the capability of such techniques to obtain structural and compositional information of the surfaces shown by these hybrid materials. Crystals of $\mathbf{5}$ were found to have surfaces with well-defined ledges formed by intersecting crystal planes with different chemical composition, as a reminiscence of the strong segregation observed in the bulk crystal structure. The fundamental (001) surface (the largest in the crystal) consists of large and narrow terraces, which expose the single PFC module $\mathbf{4}$ and the cryptate $\mathbf{3}$, respectively; while layers of HC/IS supercations and PFC superanions alternating along the $c$ crystallographic axis are exposed at the surface of the [001] zone.

The tendency of organic crystals to form well-defined ledges exposing specific molecular functionalities provides an intriguing opportunity for examining the organization of molecules on substrates for precise control of nucleation. Understanding of the physical and chemical properties of surfaces is of paramount importance for the design of novel materials. This study will prompt a rational design of new halogen-bonded PFC-HC hybrid co-crystals, leading to the subsequent synthesis of novel hybrid systems exposing engineered surfaces and exhibiting predictable functional properties. Moreover, due to the flexibility of SFM techniques and the difficulties in growing good crystals of long-chain perfluoroalkanes (because of the poor intermolecular interactions PFCs give rise to), we expect SFM to be very useful in the future as an alternative tool to X-ray diffraction methods for the investigation of such kind of highly fluorinated materials.

\section{Acknowledgements}

This work was supported by the MIUR, PRIN 2005 under project number 2005035277 and by Solvay-Solexis S.p.A. The authors wish to thank Prof. Giuseppe Resnati for useful discussions and to recognize Ms Rauni Hannele Primetta for providing technical assistance.

\section{References}

1 A. Facchetti, M. H. Yoon, C. L. Stern, G. R. Hutchison, M. A. Ratner and T. J. Marks, J. Am. Chem. Soc., 2004, 126, 13480-13501.

2 D. A. Tomalia, Nat. Mater., 2003, 2, 711-712.

3 J. G. Riess, Chem. Rev., 2001, 101, 2797-2919.

4 B. E. Smart, J. Fluor. Chem., 2001, 109, 3-11.

5 A. I. Cooper, J. D. Londono, G. Wignall, J. B. McClain, E. T. Samulski, J. S. Lin, A. Dobrynin, M. Rubinstein, A. L. C. Burke, J. M. J. Frechet and J. M. DeSimone, Nature, 1997, 389, 368-371.

6 B. E. Smart, Organofluorine Chemistry: Principles and Commercial Applications, Plenum Press, New York, 1994.

7 R. B. Walsh, C. W. Padgett, P. Metrangolo, G. Resnati, T. W. Hanks and W. T. Pennington, Cryst. Growth Des., 2001, 1, 165; P. Metrangolo and G. Resnati, Chem.-Eur. J., 2001, 7, 2511; P. Metrangolo, H. Neukirch, T. Pilati and G. Resnati, Acc. Chem. Res., 2005, 38, 386; C. M. Reddy, M. T. Kirchner, R. C. Gundakaram, K. A. Padmanabhan and G. R. Desiraju, Chem.-Eur. J., 2006, 12, 2222; P. Metrangolo, T. Pilati and G. Resnati, CrystEngComm, 2006, 8, 946; J. M. Dumas, M. Gomel, M. Guerin, in The Chemistry of Functional Groups, Supplement D, ed. S. Patai and Z. Rappoport, John Wiley \& Sons Ltd, New York, 1983, ch. 21, p. 985

8 P. Metrangolo, T. Pilati, G. Resnati and A. Stevanazzi, Curr. Opin. Colloid Interface Sci., 2003, 8, 215-222.

9 J. W. Xu, X. M. Liu, T. T. Lin, J. C. Huang and C. B. He, Macromolecules, 2005, 38, 3554-3557.

10 R. Bertani, P. Metrangolo, A. Moiana, E. Perez, T. Pilati, G. Resnati, I. Rico-Lattes and A. Sassi, Adv. Mater., 2002, 14, 1197-1201.

11 M. D. Ward, Chem. Rev., 2001, 101, 1697-1725.

12 R. S. Abendan and J. A. Swift, Langmuir, 2002, 18, 4847-4853.

13 S. Lafont, H. Rapaport, G. J. Somjen, A. Renault, P. B. Howes, K. Kjaer, J. Als-Nielsen, L. Leiserowitz and M. Lahav, J. Phys. Chem. B, 1998, 102, 761-765.

14 R. Liantonio, P. Metrangolo, T. Pilati and G. Resnati, Cryst. Growth Des., 2003, 3, 355-361.

15 C. B. Aakeröy, A. M. Beatty and B. A. Helfrich, Angew. Chem., Int. Ed., 2001, 40, 3240-3242.

16 J. Lu, E. Delamarche, L. Eng, R. Bennewitz, E. Meyer and H. J. Guntherodt, Langmuir, 1999, 15, 8184-8188.

17 K. Do, T. P. Klein, C. A. Pommerening and L. S. Sunderlin, J. Am. Soc. Mass Spectrom., 1997, 8, 688-696.

18 J. Frommer, Angew. Chem., Int. Ed. Engl., 1992, 31, 1298-1328.

19 R. M. Overney, E. Meyer, J. Frommer, D. Brodbeck, R. Luthi, L. Howald, H. J. Guntherodt, M. Fujihira, H. Takano and Y. Gotoh, Nature, 1992, 359, 133-135.

20 M. Fujihira and H. Kawate, Thin Solid Films, 1994, 242, 163-169.

21 H. Takano and M. D. Porter, J. Am. Chem. Soc., 2001, 123, 84128413.

22 M. O. Finot and M. T. McDermott, J. Am. Chem. Soc., 1997, 119, 8564-8565.

23 L. Miozzo, M. Campione, M. Moret, A. Papagni and A. Sassella, Eur. Phys. J. B, 2004, 39, 229-234.

24 H. Takano, J. R. Kenseth, S. S. Wong, J. C. O'Brien and M. D. Porter, Chem. Rev., 1999, 99, 2845-2890.

25 H. I. Kim, T. Koini, T. R. Lee and S. S. Perry, Langmuir, 1997, 13, 7192-7196. 
26 According to Stukalov, et al. [Oleg Stukalov, Chris A. Murray, Amy Jacina and John R. Dutcher, Rev. Sci. Instrum., 2008, 77, 033704, ], we typically observed a reduced sensitivity of adhesion on relative humidity ( $\mathrm{RH}$, around $40 \%$ in the AFM chamber) using cantilevers stored for long time in Gel-Packs. An histogram is provided in the electronic supplementary information for type A curves while adhesion in type B curves was almost insensitive to RH changes.

27 C. Rotsch and M. Radmacher, Langmuir, 1997, 13, 2825-2832.
28 The AFM, employing a nanometer-sized tip, probes the interaction forces on the near-atomic scale.

29 B. Bhushan, D. Hansford and K. K. Lee, J. Vac. Sci. Technol., A, 2006, 24, 1197-1202.

30 O. Noel, M. Brogly, G. Castelein and J. Schultz, Langmuir, 2004, 20, $2707-2712$.

31 N. A. Burnham, D. D. Dominguez, R. L. Mowery and R. J. Colton, Phys. Rev. Lett., 1990, 64, 1931-1934.

32 S. L. Ren, S. R. Yang and Y. P. Zhao, Appl. Surf. Sci., 2004, 227, 293-299. 